\title{
Shared Pathways Among Autism Candidate Genes Determined by Co-expression Network Analysis of the Developing Human Brain Transcriptome
}

\author{
Ahmed Mahfouz ${ }^{1,2} \cdot$ Mark N. Ziats $^{4,5,6}$ • Owen M. Rennert ${ }^{4}$.

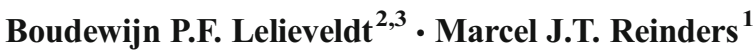

Received: 26 February 2014 / Accepted: 14 August 2015 /Published online: 23 September 2015

(C) The Author(s) 2015. This article is published with open access at Springerlink.com

\begin{abstract}
Autism spectrum disorder (ASD) is a neurodevelopmental syndrome known to have a significant but complex genetic etiology. Hundreds of diverse genes have been implicated in ASD; yet understanding how many genes, each with disparate function, can all be linked to a single clinical phenotype remains unclear. We hypothesized that understanding functional relationships between autism candidate genes during normal human brain development may provide convergent mechanistic insight into the genetic heterogeneity of ASD. We analyzed the co-expression relationships of 455 genes previously implicated in autism using the BrainSpan human transcriptome database, across 16 anatomical brain regions spanning prenatal life through adulthood. We discovered modules of ASD candidate genes with biologically relevant temporal coexpression dynamics, which were enriched for functional
\end{abstract}

Ahmed Mahfouz and Mark N. Ziats have contributed equally to this work.

Electronic supplementary material The online version of this article (doi:10.1007/s12031-015-0641-3) contains supplementary material, which is available to authorized users.

Ahmed Mahfouz

a.mahfouz@tudelft.nl

1 Delft Bioinformatics Lab, Delft University of Technology, Delft, The Netherlands

2 Department of Radiology, Leiden University Medical Center, Leiden, The Netherlands

3 Department of Intelligent Systems, Delft University of Technology, Delft, The Netherlands

4 National Institute of Child Health and Human Development, National Institutes of Health, Bethesda, MD, USA

5 University of Cambridge, Cambridge, UK

6 Baylor College of Medicine, Houston, TX, USA ontologies related to synaptogenesis, apoptosis, and GABAergic neurons. Furthermore, we also constructed coexpression networks from the entire transcriptome and found that ASD candidate genes were enriched in modules related to mitochondrial function, protein translation, and ubiquitination. Hub genes central to these ASD-enriched modules were further identified, and their functions supported these ontological findings. Overall, our multi-dimensional co-expression analysis of ASD candidate genes in the normal developing human brain suggests the heterogeneous set of ASD candidates share transcriptional networks related to synapse formation and elimination, protein turnover, and mitochondrial function.

Keywords Autism spectrum disorder · Gene co-expression network $\cdot$ Synaptogenesis $\cdot$ Mitochondrion $\cdot$ Apoptosis

\section{Introduction}

Autism spectrum disorder (ASD) is a neurodevelopmental syndrome characterized clinically by impairments in verbal and non-verbal communication, deficits in social interaction, and repetitive and/or restrictive patterns of behavior (American Psychiatric Association 2000). Despite an estimated prevalence of 1 in 88 newborns (Centers for Disease Control and Prevention 2012), and an exponential increase in recent efforts to elucidate autism neurobiology, a clear understanding of the molecular mechanisms underlying the development of ASD remains elusive. However, recent studies have firmly established a substantial role for genetic etiologies in the development of ASD. Evidence for a strong heritable risk of ASD was initially described in twin and sibling epidemiological studies of autism (Smalley et al. 1988; Ritvo et al. 1989; Steffenburg et al. 1989; Bailey et al. 1995; Hallmayer et al. 2011), and has since been firmly established through multiple 
genetic approaches (Geschwind 2011; Berg and Geschwind 2012, Krumm et al. 2014). For instance, genome-wide association studies (GWAS) (Wang et al. 2009; Weiss et al. 2009; Anney et al. 2010), copy number variation (CNV) analysis (Sebat et al. 2007; Szatmari et al. 2007; Marshall et al. 2008; Pinto et al. 2010; Levy et al. 2011; Sanders et al. 2011), and whole-exome sequencing projects (O'Roak et al. 2012; Sanders et al. 2012; Iossifov et al. 2012; Neale et al. 2012; De Rubeis et al. 2014; Iossifov et al. 2014) have implicated hundreds of genes in ASD. Yet, understanding how this diverse set of genes relates to the underlying molecular mechanisms and subsequent neuropathology of ASD is still unclear.

Mechanistic understanding of how ASD candidate genes relate to the neurobiology of autism is a difficult task, since genes encode multiple highly complex functions at different stages of development and across different regions of the brain. Moreover, the set of genes implicated in ASD is highly heterogeneous, and many of their functions are completely unknown. Furthermore, understanding how disruption in different genes with disparate functions still results in a common clinical phenotype makes developing common targeted biomarkers and treatments for ASD challenging. Therefore, in addition to attempts to identify genes that are causative for ASD, it is important to understand how ASD candidate genes may relate to each other during human neurodevelopment in order to identify potential shared molecular pathways.

One validated approach to integrate heterogeneous gene sets, in order to uncover shared molecular mechanisms, is through the analysis of gene co-expression patterns, which invokes the guilt-by-association heuristic that is pervasive in genomics research (Stuart et al. 2003; Wolfe et al. 2005). Several studies have demonstrated that genes with similar brain co-expression patterns are likely to function together in common cellular pathways (Oldham et al. 2008; Winden et al. 2009). These transcriptional co-expression relationships are particularly relevant to neurodevelopment, as the precise regulation of gene expression across brain regions at different ages instructs the exquisite specialization and connectivity within the brain. Since neurodevelopmental disorders such as autism are believed to result from functional aberrations within brain regions and/or disruption of inter-regional connectivity between regions (Geschwind and Levitt 2007), investigating the gene expression profiles of autism candidate genes across brain regions and throughout normal human neurodevelopment may provide insight into the complex functional genomics of this neurodevelopmental disorder.

A global survey of ASD gene co-expression patterns across normal human neurodevelopment could therefore facilitate our translation of ASD candidate genes to ASD candidate pathways, but this has not yet been undertaken. A recent study that assessed autism gene co-expression patterns in two adult human brains is an important step toward this goal (Ben-David and Shifman 2012b), but as autism is a neurodevelopmental disorder, it is imperative to understand the relationship of autism candidate genes in a developmental context. Conversely, other studies have explored the expression profiles of individual ASD candidates in human brain development (Kang et al. 2011) but lack an assessment of the relationships among these ASD candidates and how they relate to global transcriptional pathways important in brain development.

Transcriptome-based studies of the developing human brain have previously been limited in the sample size, number of brain structures analyzed, and developmental time points assessed, hampering the ability to evaluate the genetic contributors to neurodevelopmental disease comprehensively (Sun et al. 2005; Abrahams et al. 2007; Johnson et al. 2009; Ip et al. 2010; Somel et al. 2010). However, the recent availability of broad developmental surveys of gene expression, which cover many brain regions over multiple developmental stages, can greatly facilitate such analysis (Kang et al. 2011). The BrainSpan Transcriptional Atlas of the Developing Human Brain is a repository of RNA-seq expression profiling of 16 brain structures spanning early pre-natal development ( 8 weeks postconception) to adulthood (40 years of age). This publicly available atlas presents a unique opportunity to understand the spatial and temporal specificity of ASD candidate genes.

A few studies have recently assessed for co-expression relationships between subsets of autism-related genes and/or certain developmental windows using human brain gene expression relationships. For instance, Parikshak et al. analyzed the co-expression of autism and intellectual disability risk genes in neocortex and among cortical laminae from samples representing early development using weighted gene coexpression network analysis (WCGNA). They demonstrated that ASD risk genes were enriched in modules related to transcription and synaptic development, and furthermore that enriched in superficial cortical layers and glutamatergic projection neuron (Parikshak et al. 2013). Willsey et al. studied co-expression networks derived from nine genes harboring recurrent de novo loss-of-function mutations in autism patients and showed principally that the autism risk gene expression is most prominent in layer $5 / 6$ cortical projection neurons during mid-fetal gestation (Willsey et al. 2013). Finally, using a different computational approach, Hormozdiari et al. integrated co-expression networks and protein-protein interaction networks of autism and intellectual disability risk genes identified in a recent cohort of 116 patients, and also showed that the autism genes enrich into networks related to transcription and synaptogenesis (Hormozdiari et al. 2015). Despite the importance of these results and their largely overlapping findings, no study has yet assessed very broad sets of autism risk genes across all brain regions and development time points to gain insight into potentially shared molecular pathways or affected brain regions among the incredibly heterogeneous autism genetic subtypes. 
Here, we present an analysis of the spatial-temporal coexpression of ASD candidate genes across the normal developing human brain using the BrainSpan atlas. We developed a biologically driven computational approach to deduce functional relationships among this diverse set of genes. We first discovered modules of ASD candidates with biologically relevant temporal co-expression dynamics. These modules were related to the processes of synaptogenesis, apoptosis, and the neurotransmitter $\gamma$-aminobutyric acid (GABA). Then, we created a transcriptome-wide co-expression network from all genes expressed in the brain, to discover significant "molecular interaction modules" and demonstrated that ASD candidate genes are enriched only in modules related to the processes of synaptogenesis, mitochondrial function, protein translation, and ubiquitination. Lastly, we identified hub genes within the ASD-enriched molecular interaction modules, whose functions supported our ontological results, and which may serve as additional ASD candidate genes. Our analysis of this multi-dimensional expression data suggests pathways previously independently implicated in autism are related to each other through shared neurodevelopmental transcriptional networks.

\section{Results}

\section{Spatio-temporal Gene Co-expression Analysis of ASD Candidate Genes}

In order to identify functional relationships between ASD candidate genes, we investigated patterns of gene coexpression change across developmental stages between each pair of genes from the ASD list. First, the correlation between each pair of ASD genes was calculated separately within each developmental stage based on the Spearman's rank correlation between the two genes across all brain regions. For each gene pair, this resulted in a correlation value for each of the seven developmental stages, representing the brain-wide transcriptional similarity between the genes at each developmental stage (Fig. 1c, d). Gene pairs were retained only if they had an absolute correlation value greater than 0.8 in at least one developmental stage. We have used the Spearman's rank correlation as it focuses more on the similarity in the change of gene expression; as opposed to similarity in the absolute values of gene expression (see the Supplementary Information for more details).

Second, the surviving gene pairs were hierarchically clustered into distinct modules based on the similarity of their correlation profiles over time (using the Euclidean distance between the profiles and a complete linkage to merge clusters). Finally, the correlation pattern for each module was summarized by averaging all the gene pair correlation patterns included in the respective module. It is worth noting that the patterns within the modules represent changes in coexpression across development (which should not be confused with actual expression levels of genes).

\section{ASD Gene Modules Display Distinct Temporal Dynamics Around Birth}

Figure 2a shows the hierarchical clustering of the retained ASD gene pairs. In total, there were 103,285 pair-wise correlations between the $455 \mathrm{ASD}$ candidate genes in the ASD list, of which 1168 remained after applying the stringent threshold of an absolute correlation greater than 0.8. The surviving gene pairs clustered into three distinct modules. Two of these modules, the "green" module and the "blue" module, displayed distinct correlation patterns relative to pre- versus postnatal development. The green module (Fig. 2b) consisted of gene pairs that lose correlation in the middle stages of development (infancy and childhood); that is, each pair of genes within the green module has highly correlated spatial expression profiles at prenatal developmental stages, but this correlation is lost at birth. In contrast, the blue module (Fig. 2c) consisted of gene pairs that gain correlation during development. These genes do not show correlation at prenatal stages but progressively increase correlation throughout postnatal development. The "red" module did not show any coordinated pattern of expression over developmental time (Fig. S1). Genes forming gene pairs in each of the three modules are listed in Table S1.

Fig. 1 Analyzing ASD candidate genes in the BrainSpan Atlas. a Temporal description (i.e., age points) of the number and sex of the assessed brains. The data were grouped into seven developmental stages based on age. Black-colored brains indicate male donors, and red-colored brains indicate female donors. b A representation of the 16 structures sampled in the BrainSpan Atlas. c Each heatmap shows the expression of all genes across six representative brain regions (AMY, HIP, STR, MD, CBC, and NCX) in three representative developmental stages. The ASD list was created by combining lists of ASD candidate genes from three sources (AutDB, AGD, and AutKB-484). d A co-expression network of ASD candidate genes was generated for each developmental stage by correlating the expression vectors across brain regions. The correlation between each gene pair was tracked over the developmental stages. The blue gene pair represents two genes that are moderately correlated at early developmental stages but gain correlation through development. Stronger correlation is represented by a thicker edge between the two nodes. By contrast, the red gene pair represents two genes that lose correlation over development. Bottom, the correlation patterns of all gene pairs in the network (gray) across development. Correlation patterns of the blue and red pairs are shown in respective colors. Birth and the average age of ASD diagnosis are indicated. e The transcriptome-wide molecular interaction network was constructed based on the pairwise correlation between each pair of genes expressed in the BrainSpan Atlas (13,563 genes). Each node in the network represents a gene while the weighted edges represent correlations between genes based on their expression across all samples. Nodes were clustered into modules (dashed circles). Genes from the ASD list are highlighted within each module (blue nodes). Blue circles indicate modules that are significantly enriched in genes from the ASD list 
a

30 "normal" donors

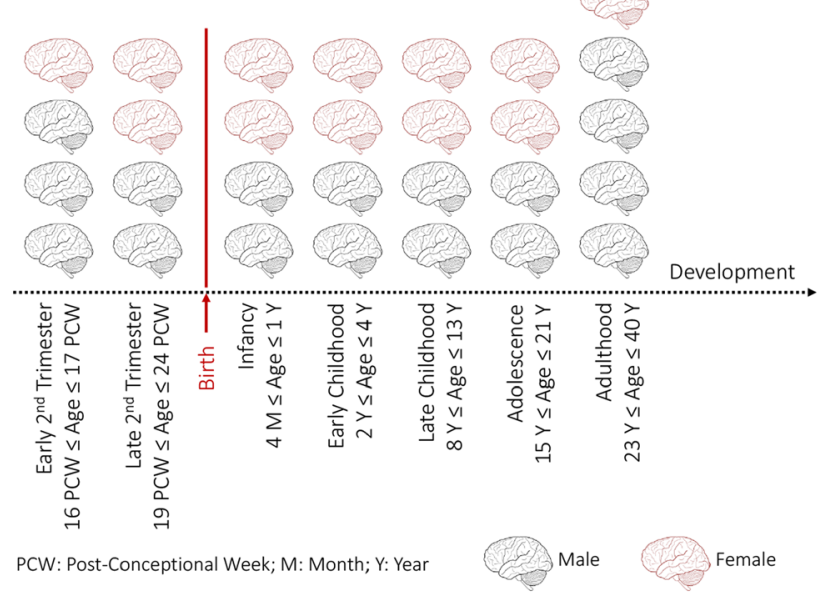

b

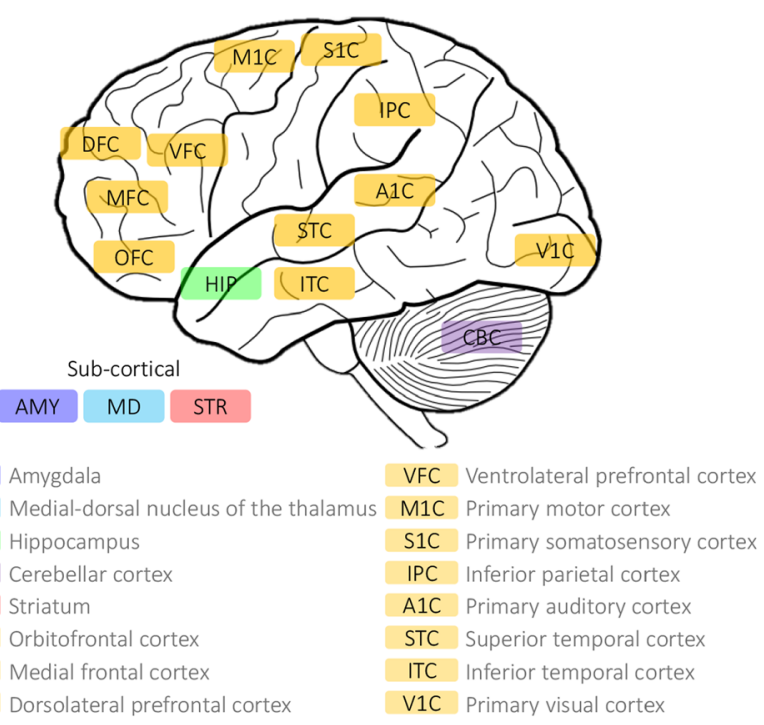

AMY Amygdala

MD

HIP Hippocampus

CBC Cerebellar cortex

STR Striatum

OFC Orbitofrontatcortex

MFC Medial frontal cortex

DFC Dorsolateral prefrontal cortex

C

d

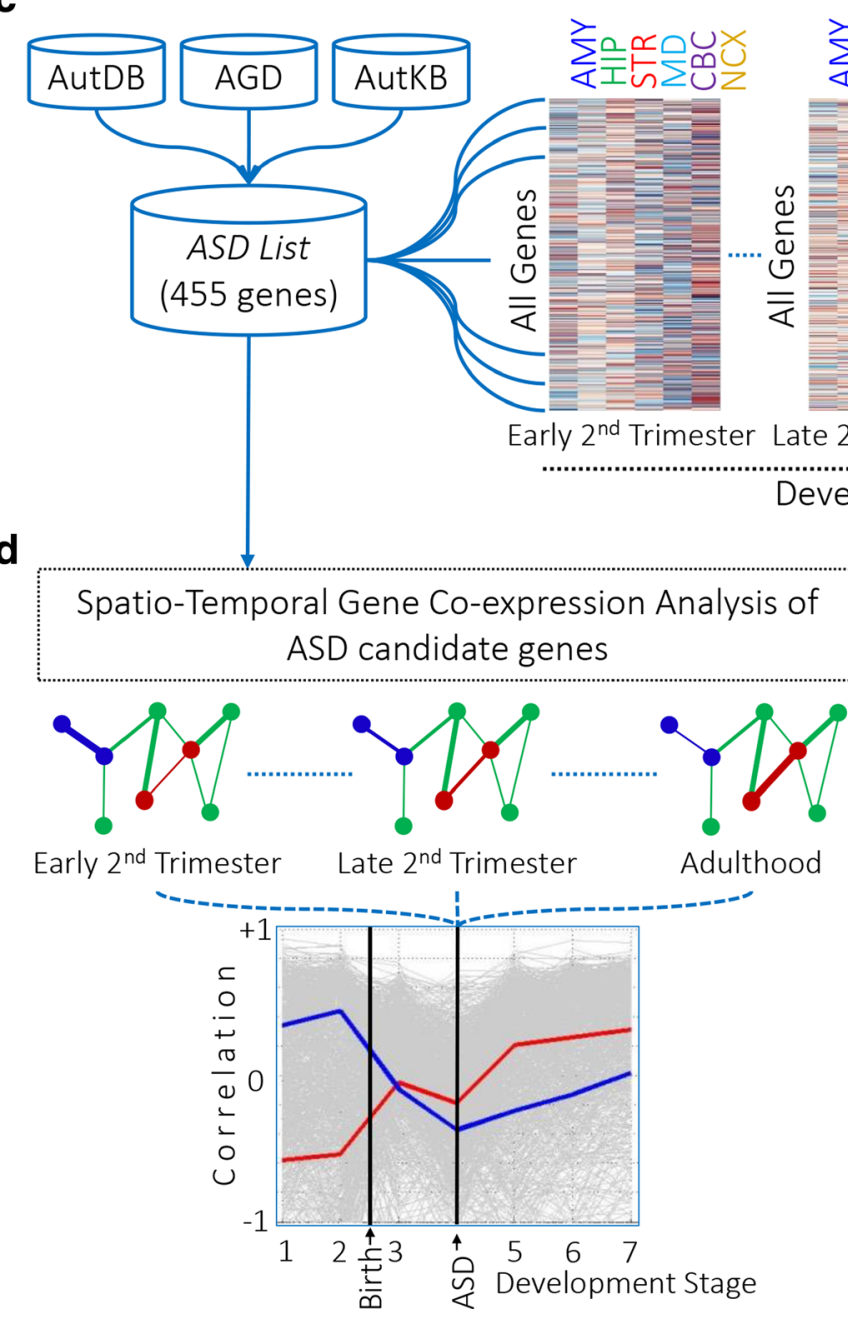

c 
To characterize these modules further, we used the gene ontology (GO) enrichment analysis tool DAVID 6.7 (Huang et al. 2009) to discover whether genes in these modules relate to specific molecular mechanisms, cellular pathways or disease annotation terms. The top significantly enriched terms (Benjamini-corrected $p$ values $<0.01$ ) are summarized as shown in Fig. 2d. All the three modules were enriched for annotation terms related to neuron projection, synapse, synaptic transmission, and behavior. The three modules were also enriched for disease terms including mental retardation and epilepsy. The green and blue modules were significantly enriched for neuron differentiation, cell morphogenesis, and learning/memory. The green module was specifically enriched in functional terms related to regulation of apoptosis and regulation of cell death, while the blue module was specifically enriched in terms related to ion channel, neurotransmitter receptor activity, and GABA receptor activity. Table S2 includes the full list of enriched gene-annotation terms for these two modules.

None of the GO terms that were significantly enriched in the three ASD modules showed any significant enrichment in modules from ten randomly created sets (see Table S3). We also assessed how many gene pairs remained after thresholding them on co-expression (absolute correlation $>0.8$ at any developmental stage) in 10,000 random gene sets of 455 genes. The results are summarized in Fig. S2, where we show that the number of gene pairs remaining after thresholding the ASD list (1168 gene pairs) is highly significant $\left(p<10^{-4}\right)$.

\section{Modules of ASD Candidate Genes Are Enriched in Neurons}

We then assessed if these modules were enriched in specific brain cell types. Lists of cell-type-specific genes were obtained from a previously published work (Cahoy et al. 2008). These lists included 1465 neuron-, 1529 oligodendrocyte-, and 1829 astrocyte-specific genes (Table S4). ASD candidate gene modules were assessed for enrichment of these cell types using the hypergeometric probability test (see "Material and Methods"). Both the green and blue modules were significantly enriched in neurons, whereas the red module demonstrated no significant enrichment, as shown in Fig. 2e.

\section{Enrichment of ASD Candidate Genes in Transcriptome-Wide Molecular Interaction Modules}

Given the marked genetic heterogeneity of ASD and the large number of genes involved, it is also important to understand the role of ASD candidate genes in normal brain development within the context of the whole transcriptome, as subnetworks of the entire brain transcriptome may be perturbed by the ASD candidates. An analysis of these subnetworks could reveal
ASD-related pathways that would be missed by analyzing the ASD candidates alone, as it is unlikely that all ASD candidate genes have been identified to date (Sanders et al. 2012). Moreover, this top-down approach allows the identification of other genes that might also relate to ASD. Therefore, we performed a transcriptome-wide co-expression network analysis to identify functionally related gene modules throughout the normal developing brain transcriptome ("molecular interaction modules"). Then, we assessed whether these modules were specific to distinct brain regions or developmental stages, and if they were related to specific pathways, cellular processes, or disease annotation terms. Finally, we determined if ASD candidate genes were enriched in any of the resultant molecular interaction modules.

\section{No Evidence for Region-Specific Modules}

The transcriptome-wide co-expression network was constructed from all genes expressed in the brain (13,563 genes), based on their expression profile across all samples (480 samples, i.e., all brain structures and developmental stages). Genes were hierarchically clustered based on Spearman's rank correlation and complete linkage between pairs of genes. The resulting network consisted of 32 modules of varying size (from 36 to 1386 genes), as shown in Fig. 3a. Visual analysis of the heatmap and average expression patterns of member genes from each of the 32 modules demonstrated that none were specific to particular anatomical regions. This observation is consistent with the results from a similar dataset of human brain development assessed by microarray (Ben-David and Shifman 2012a). We did not observe any pre/postnatalspecific expression patterns in any of the 32 modules (Fig. S3). The genes comprising each of the 32 modules are listed in Table S5.

\section{Modules Enriched for ASD Genes Relate to Synaptogenesis, Protein Turnover, and Mitochondria}

The resulting transcriptome-wide co-expression modules were then assessed for enrichment of genes belonging to the ASD list using the hypergeometric probability test. Four modules - magenta, brown, orange, and purple - were significantly enriched for ASD candidate genes (FDR-corrected $p$ values $<0.001$ ), as shown in Fig. 3b. The magenta module (Fig. 4a) contained highly co-expressed genes during early childhood. The brown module (Fig. 4b) included genes with weak coexpression during childhood and differential spatial coexpression at late developmental stages. The orange module (Fig. 4c) contained genes with progressively increasing coexpression during development. Finally, the purple module (Fig. 4d) included genes with varied co-expression during development and high differential spatial co-expression in adolescence and adulthood. 
a

Early $2^{\text {nd }}$ Trimester

Late $2^{\text {nd }}$ Trimester

Infancy

Early Childhood

Late Childhood

Adolescence

Adulthood

Correlation

d

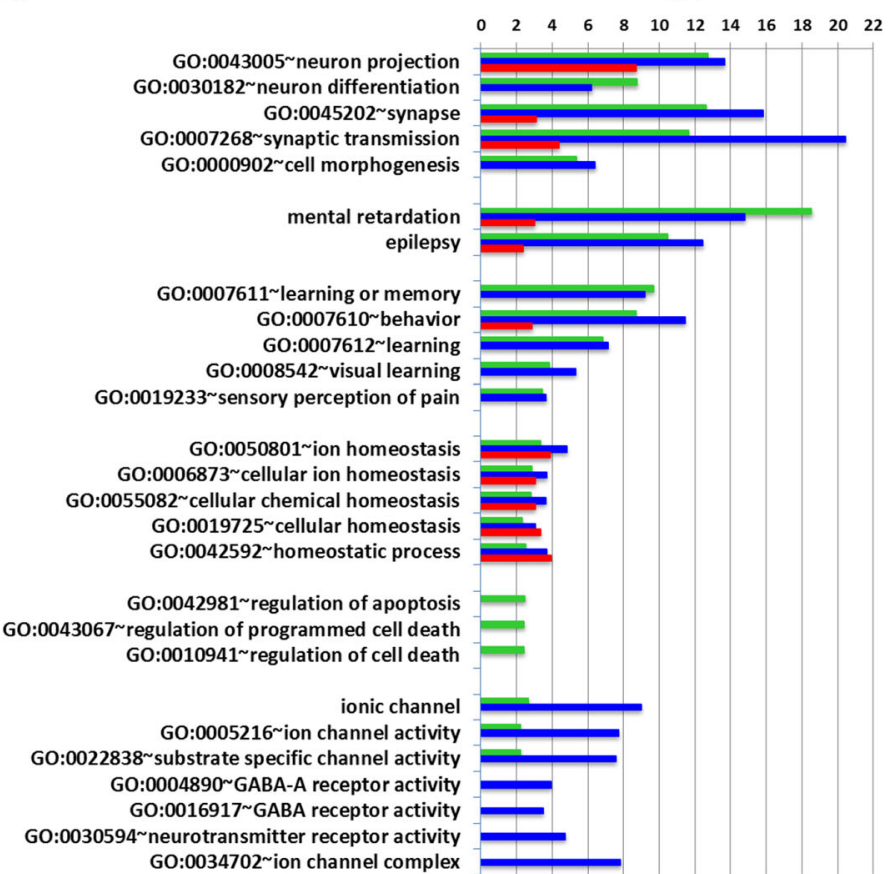

b

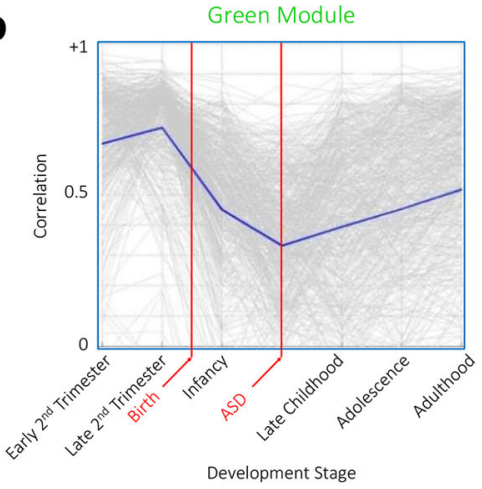

C

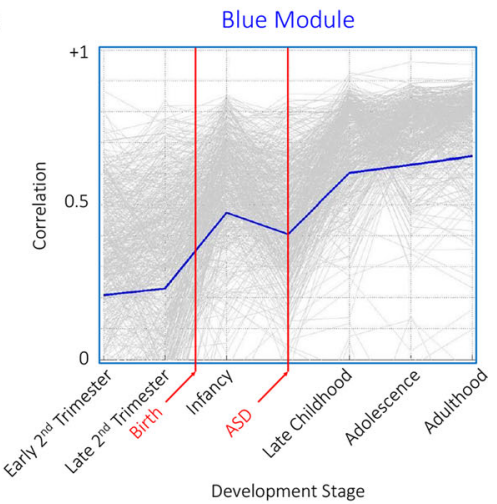

e

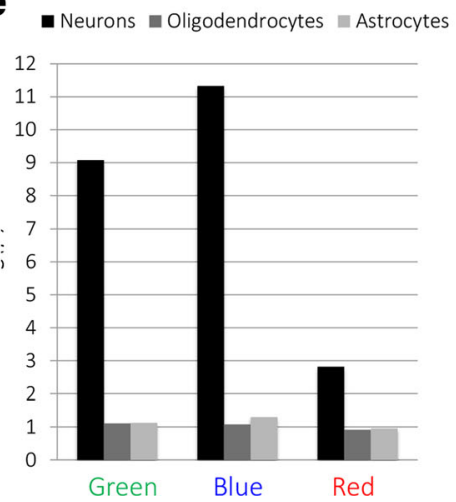

Fig. 2 Spatio-temporal gene co-expression analysis of ASD candidate genes. a Heatmap of the temporal correlation pattern of ASD gene pairs (rows) through different developmental stages (columns). The dendrogram to the right shows the clustering of ASD gene pairs into three modules (red, green, and blue). b The average correlation pattern of gene pairs in the green module shows loss of correlation at childhood. Vertical lines indicate birth and average age of ASD diagnosis. $\mathbf{c}$ The average correlation pattern of gene pairs in the blue module shows progressive gain of correlation across development. d Gene ontology terms enriched in each of the three modules (represented in $-\log _{10}(p)$, Benjamini-corrected). Bars are colored according to the module's name. e Enrichment scores for each of the ASD modules in neurons, astrocytes, and oligodendrocytes (represented in $-\log _{10}(p), F D R$-corrected)

the purple module. Enrichment of ASD candidate genes into transcriptome-wide synapse modules further supports our previous finding of ASD modules (green and blue modules), above, which were also related to synaptogenesis. Neurological disease terms were also significant in the ASD-enriched modules: epilepsy (brown module), Parkinson's (magenta and orange modules), Alzheimer's (magenta and orange modules) and Huntington's (magenta and orange modules). 


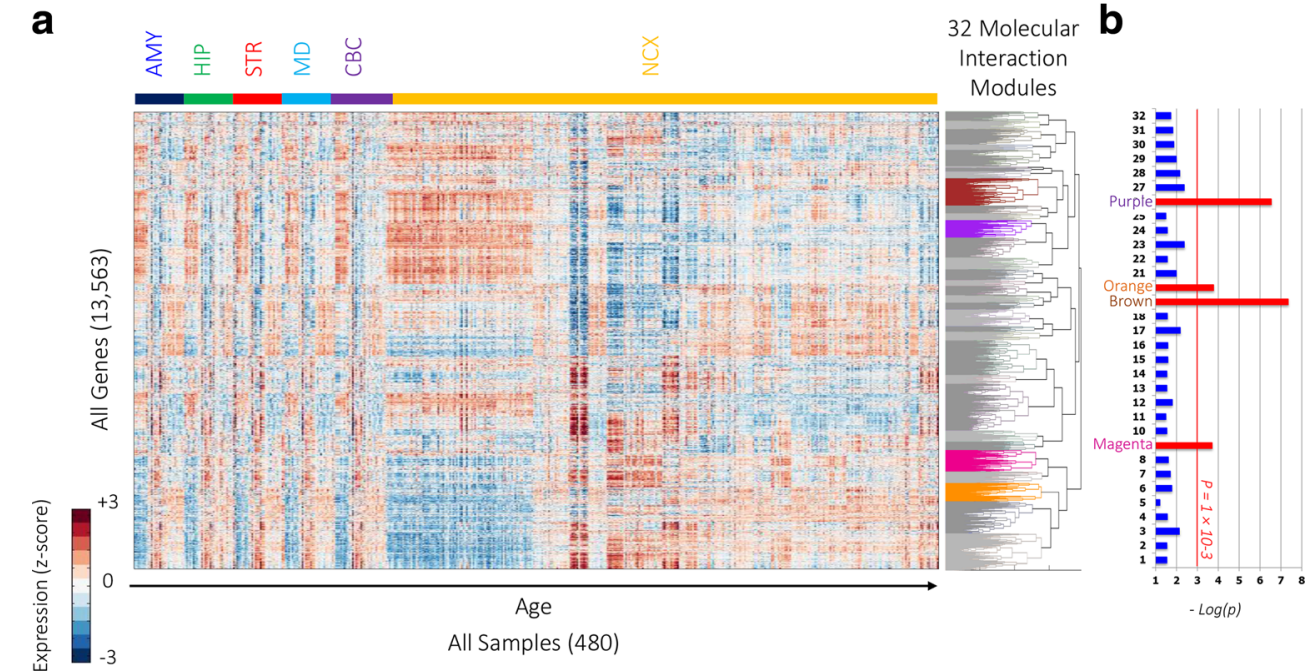

b

Fig. 3 Transcriptome-wide molecular interaction network. a A heatmap of the expression of 13,563 genes (rows) across all 480 samples (columns). Samples are ordered first by brain region (color-code at the top) and then by age. The dendrogram to the right shows the clustering of all the genes into 32 modules. Modules with significant enrichment

$\left(p<10^{-3}\right)$ of genes from the ASD list are colored while other modules are shown in gray. $\mathbf{b}$ Enrichment of ASD candidate genes in each of the modules showing high significance in the magenta, brown, orange, and purple modules (represented by $-\log _{10}(p), F D R$-corrected)

\section{ASD-Enriched Molecular Interaction Modules Are Mainly Neuronal}

Each module was also tested for enrichment of specific neural cell populations (i.e., neurons, oligodendrocytes, and astrocytes), as described earlier. Three out of the four ASDenriched modules were enriched for neurons (magenta, brown, and purple modules), as shown in Fig. 5. The orange module, which was related to mitochondrial functioning, was highly enriched in astrocytes but not neurons. This finding is of relevance, as multiple recent studies have implicated glia, and specifically astrocytes, in the brain pathology of autistic subjects (Lioy et al. 2011; Cao et al. 2012).

\section{ASD-Enriched Molecular Interaction Module Hub Genes Provide Molecular Targets}

An alternative approach to annotate the function of each ASDenriched module is to analyze the genes with the strongest correlations within each module. It has been shown that within an interaction network, genes with the most connections to other genes, termed hub genes, are informative for the network as a whole, and are potential high-yield therapeutic targets (Barabási et al. 2011). The strongest correlations within a module were explored using Cytoscape v2.8 (Smoot et al. 2011). First, each ASD-enriched module (magenta, brown, orange, and purple) was imported as a graph with genes acting as nodes and pair-wise correlations between genes representing edges between the nodes. Figure 6 shows a subset of the connected nodes within each graph.

The ten most highly connected nodes (genes) within each graph were extracted and their putative functions determined by manual curation of the literature. Among these most highly connected hub genes, a number were of note. The most striking observation was that most of the highly connected hub genes in the magenta and brown modules are known to function in the processes of chromatin remodeling, transcription, or translation (HMGN3, EIF3K, ZFAND6, DNAJC1, C6orf130, ERCC1, LCMBT2, MBTPS2, KIAA1191, C14orf138, GDA, and NCOA7). This result is in line with the gene ontology enrichment for these modules (Fig. 4). A number of other central hub genes are involved in intracellular signaling pathways (PROCA1, TBC1D22B, PPP2R2D, $H A C E 1)$ and a few are known to function as membrane ion channels (PRRT1, KCTD4, SLC26A1, KCNA4). In addition, a number of hub genes function in apoptosis or myeloid/ microglia cell processes (such as RNF11, CD200, and $F A F 1$ ). These hub gene functions largely recapitulate the ontologies of their respective networks, supporting our enrichment results and highlighting potential critical regulatory molecules of these networks.

\section{Discussion}

In order to gain insight into the molecular pathogenesis of ASD, we present a biologically driven computational approach to analyze a heterogeneous set of genes previously independently implicated in ASD, to understand if they may relate to each other through shared functional genomics mechanisms. The main goal of this work is to understand if ASD 

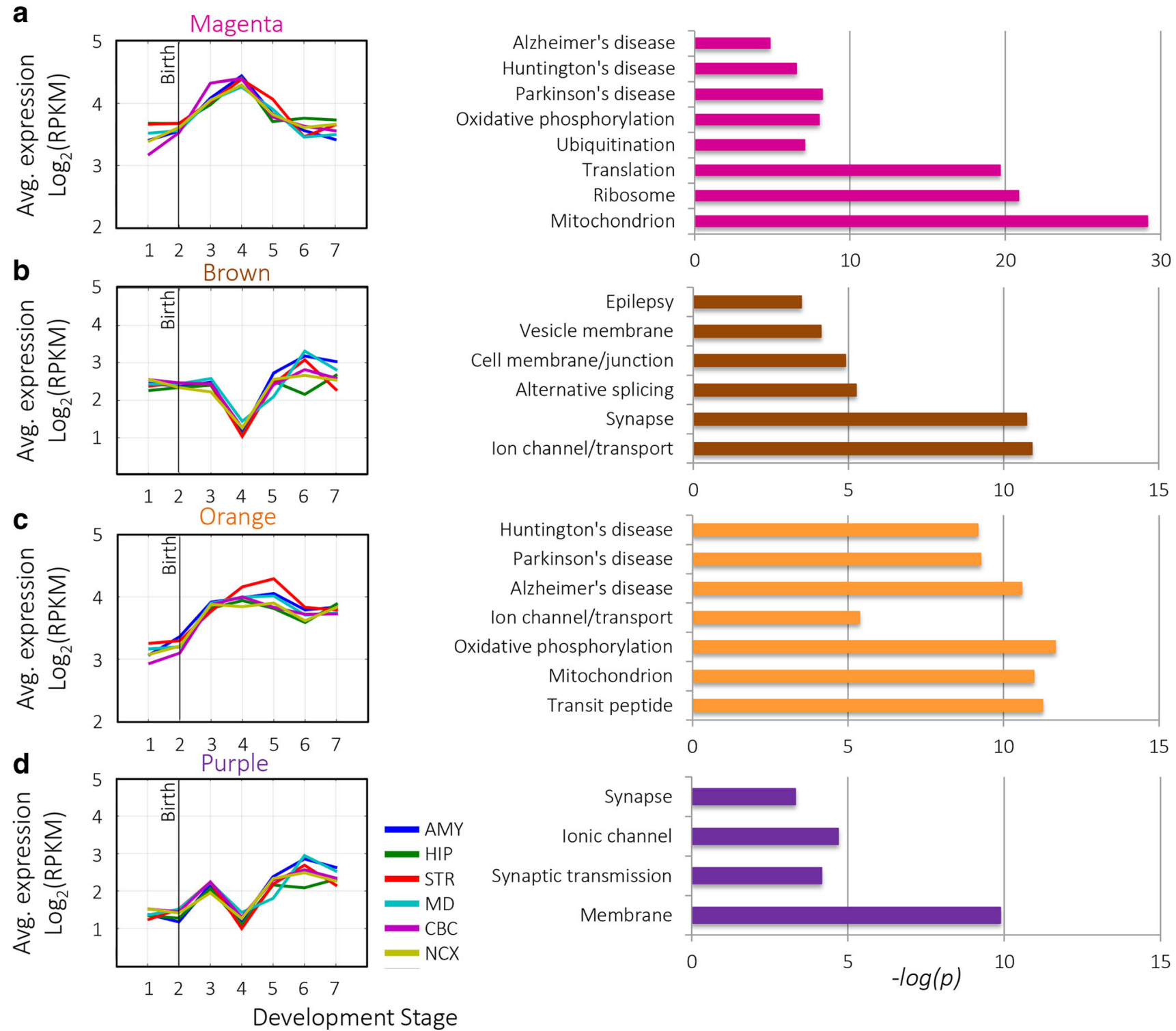

Fig. 4 ASD modules. a Left, average expression pattern of the magenta module genes across different brain regions (different plot colors). Right, top GO terms enriched in the magenta module. b Left, average expression pattern of the brown module genes. Right, top GO terms enriched in the brown module. c Left, average expression pattern of the orange module

genes. Right, top GO terms enriched in the orange module. d Left, average expression pattern of the purple module genes. Right, top GO terms enriched in the purple module. All enrichment values are represtented by $-\log _{10}(p)$, Benjamini-corrected

candidate genes relate to common cellular/molecular pathways when considered in the context of transcription during normal human brain development. Identifying such pathways has profound implications for understanding the pathophysiology of ASD, especially since the majority of ASD patients do not have an identifiable genetic mutation (Huguet et al. 2013). Yet, those patients are still likely to have alterations in the same pathways that are affected as those ASD patients with genetic mutations, although the alterations may be caused by environmental, epigenetic, or other non-genetic factors.

We intentionally analyzed a very broad collection of genes associated with ASD, in an attempt to understand if there are

cellular or molecular pathways that may represent final common mechanisms across all patients. Despite the fact that some of the genes in our ASD list are essentially causative for ASD (for instance, single gene mutation syndromes such as Fragile X), while others are not as strongly associated, we have weighted all genes equally to avoid bias toward more severely affected patient cases. Future work could attempt to weigh genes differently within the co-expression networks to study different genetic subtypes of autism.

We discovered subsets of ASD candidate gene modules that displayed biologically relevant co-expression dynamics, which were enriched for the processes of synaptogenesis, apoptosis, and GABA-ergic signaling. In addition, we assessed 
Neurons Oligodendrocytes Astrocytes

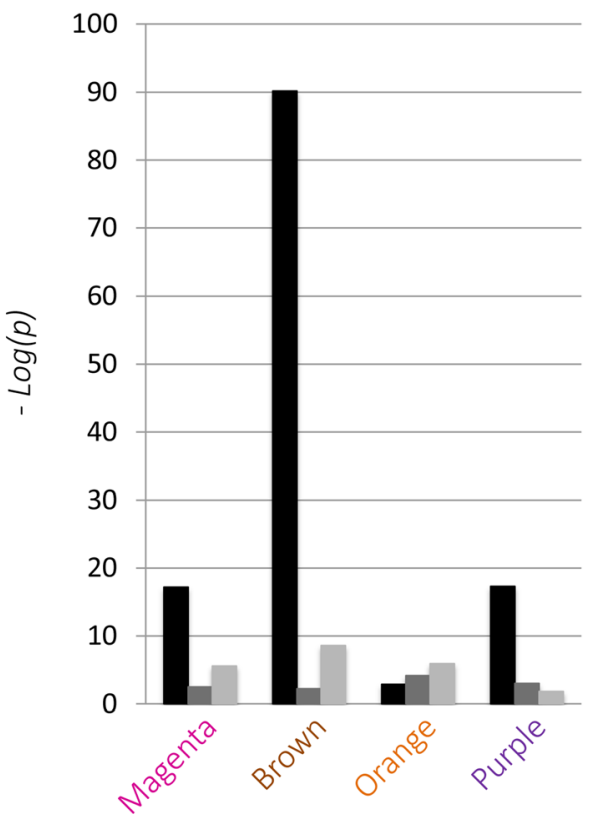

Fig. 5 Enrichment of the ASD modules in cell-type-specific genes. Enrichment of ASD-enriched modules in neurons, oligodendrocytes, and astrocytes (represented in $-\log _{10}(p), F D R$-corrected)

for functional genomic relationships between ASD candidate genes and the entire developing human brain transcriptome. This analysis revealed that ASD candidate genes are enriched within transcriptome-wide modules related to synaptogenesis, mitochondrial function, alternative splicing, protein translation, and ubiquitination. By identifying gene modules that have similar expression patterns in the brain (regardless of time period), we were able to infer that they are likely functioning in similar pathways. This allowed us to infer which cellular and molecular mechanisms are likely to be disrupted in autism. We also demonstrated the cell-type-specific enrichment of these modules being mostly neurons, further supporting the biological relevance of our computational approach, as the broad ASD phenotype is generally consider to ultimately result from neuronal/synaptic abnormalities (Zoghbi 2003). Although several brain regions have been highlighted in neuroimaging and connectivity studies of autistic brains (namely cortical regions and the cerebellum) (Carper and Courchesne 2005; Courchesne and Pierce 2005), interestingly, none of the transcriptome-wide modules were specific to particular anatomical regions, which supports previous reports of the BrainSpan dataset via microarray (Kang et al. 2011). Finally, by assessing genes with the highest connectivity within the transcriptome-wide molecular interaction modules that were enriched for ASD candidates, we identified hub genes that may represent critical regulatory molecules in these networks, and their functions further supported our enrichment findings.
The number of strongly connected gene pairs from the ASD list were found to be highly significant $\left(p=10^{-4}\right)$, indicating that — based on their significantly strong co-expression across development - those ASD-associated genes are likely to be functionally related. We discovered three subsets of ASD-associated genes with distinct co-expression profiles around birth, even though the co-expression network for each developmental stage was calculated separately to avoid any bias toward pre/postnatal expression changes. All three of these modules were significantly enriched for the processes of synaptogenesis and behavior, in addition to the disease annotations of mental retardation and epilepsy. Two of the modules (the green and blue modules) were also significantly associated with cell morphogenesis, neuron differentiation, and learning. Moreover, the green module, which had highly correlated spatial expression at prenatal developmental stages with a dramatic loss of correlation at birth, was uniquely enriched for the process of apoptosis. Conversely, the blue module displayed an opposite co-expression trajectory - poor correlation in expression prior to birth, but strong coexpression beginning in infancy and increasing through adulthood - and was uniquely related to GABA-ergic signaling and ion channels. The distinct, biologically relevant expression patterns of these two modules around birth, a developmental period with the greatest shifts in gene expression (Kang et al. 2011), suggests a key role of these networks in brain development and autism.

ASD-associated genes were highly co-expressed later in development in some of the identified modules (childhood and adulthood), whereas autism symptoms are generally apparent by the age of two. Our results suggest that a heterogenous set of genes which were independently associated to ASD converge into few functional pathways late in normal development. However, our findings do not preclude the possibility that the pathways implicated by these modules are involved in ASD pathogenesis, as our analysis was on coexpression patterns, not absolute gene expression levels. It is possible that the genes in these modules are still expressed in early neurodevelopment but that they are most strongly coexpressed with other genes in the same module later in life. Consequently, disruption of the integrity of these genes (through inherited mutations, de novo mutations, mis-expression, etc.) early in development is likely to disrupt the functions of those modules later in life.

The functional ontologies of these networks are all pathways previously implicated ASD. Disrupted synaptogenesis has been one of the most replicated findings in ASD research (Bourgeron 2009), and autism is largely considered to be a disorder that results from a convergence of factors into synaptic dysfunction (Zhoghbi 2003). Our finding of multiple ASD gene co-expression networks enriched for the function of synaptogenesis is in line with these previous studies. Additionally, our analysis shows these same transcriptional networks are 

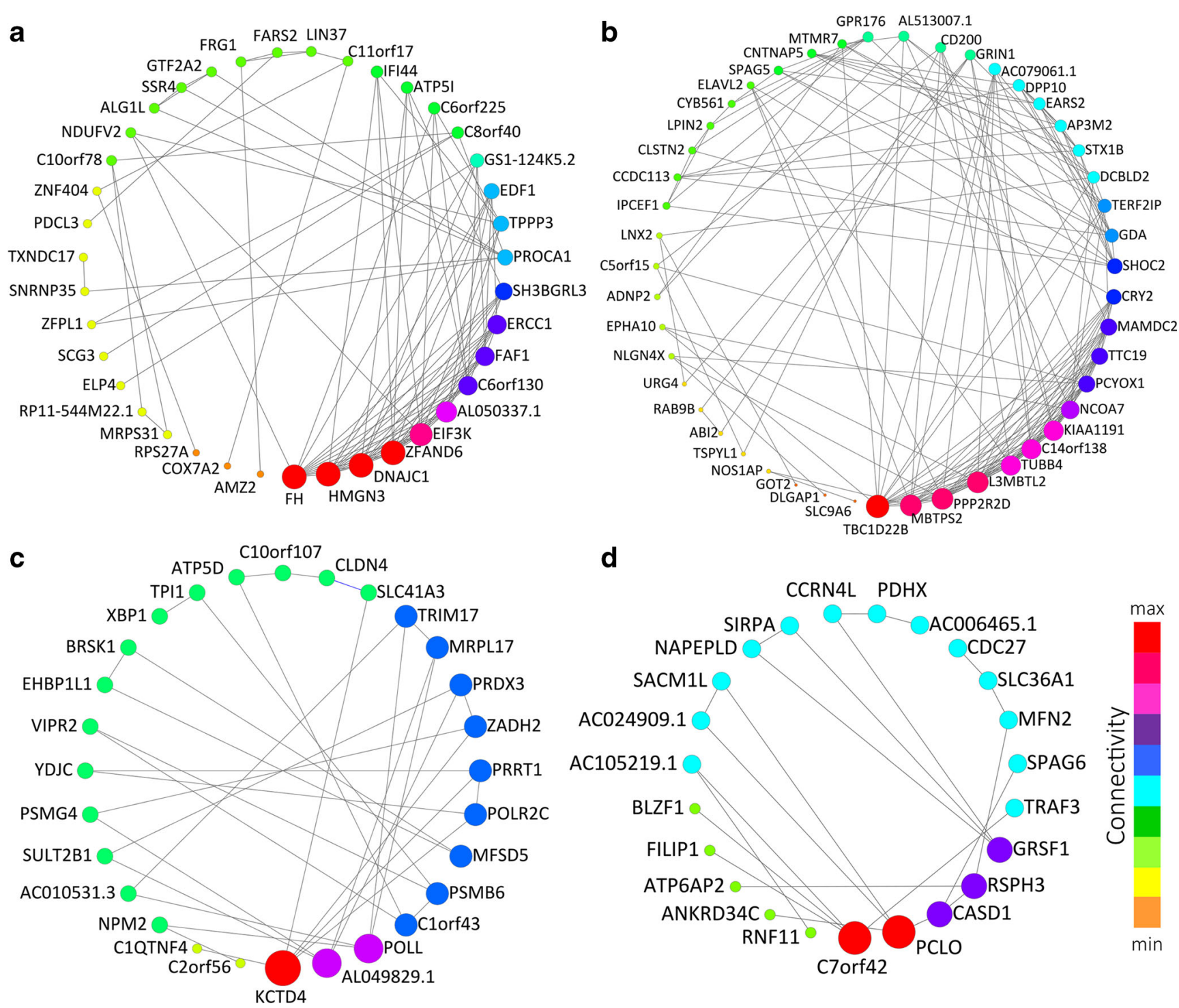

Fig. 6 Hub genes of ASD modules. Each of the four ASD-enriched modules is presented with the degree-sorted circle layout of cytoscape, with the nodes' size and color reflect the level of connectivity within the network. The bigger the node, the more connections it has. For clarity,

edges with correlation values smaller than 0.9 are removed. a Topconnected genes of the magenta module. $\mathbf{b}$ Top-connected genes of the brown module. c Top-connected genes of the orange module. d Topconnected genes of the purple module

related to the processes of GABA-ergic signaling and apoptosis, which have been independently associated with ASD through various approaches. GABA-ergic neurons are the main inhibitory cells of the brain, and much research has suggested that an imbalance in the ratio of inhibitory to excitatory neurons may underlie autism at the cellular circuit level (Rubenstein and Merzenich 2003). Furthermore, a number of clinical trials are currently ongoing to test GABA-ergic modulators for the treatment of ASD (Spooren et al. 2012). Likewise, apoptosis - and more specifically the pruning of overabundant neural connections in early development-has recently been shown to be a critical process in the developing mammalian brain (Paolicelli et al. 2011), and a number of studies have suggested this process may be aberrant in ASD
(Sheikh et al. 2010; Maezawa et al. 2011). A delicate balance between formation of needed synaptic connections and pruning of overabundant connectivity (and their excitatory/ inhibitor ratio) is a main component of early experiencedependent brain development, and both human and animal studies have previously shown deficiencies in these processes in ASD (Courchesne and Pierce 2005). Our results suggest these processes may relate to each other and to ASD candidate genes through shared transcriptional networks.

ASD candidate gene modules with distinct temporal coexpression profiles around birth, which are highly related to synaptogenesis, support the notion that the pathogenesis of ASD is strongly related to this process. Additionally, the demonstration that the same transcriptional networks are also 
related to GABA-ergic signaling and apoptosis-both also suggested to be aberrant in autism - suggests that these disparate pathways may relate to each other through underlying shared transcriptional networks, providing a potential mechanism for functional convergence of ASD candidate genes into common pathways underlying autism.

By incorporating the ASD candidate genes into the context of the entire brain transcriptome, our results suggest that the disruption of synaptogenesis in autism may also relate to underlying basic cellular processes - alternative splicing, protein translation, and ubiquitination - which have previously been implicated in ASD (Kelleher and Bear 2008; Glessner et al. 2009; Smith and Sadee 2011; Piton et al. 2012). Defects in protein translation in particular have recently been shown to be a prominent feature in multiple animal models of ASD (Neves-Pereira et al. 2009; Gkogkas et al. 2013; Santini et al. 2013).

Two transcriptome-wide modules that were enriched for ASD candidate genes were both related to mitochondrial function, and one was specifically enriched in glia but not neurons. A large body of evidence has associated mitochondria dysfunction with rare syndromic forms of autism (Rossignol and Frye 2012) and recent evidence suggests that altered mitochondrial gene expression may contribute to non-syndromic autism as well (Anitha et al. 2012a, b). Furthermore, these modules were also related to Huntington's and Alzheimer's disease, both known to have mitochondrial defects associated with their pathogenesis (Sheng and Cai 2012). While the ASD-only gene modules in the first part of this study did not implicate mitochondrial function, significant enrichment of ASD genes in two different transcriptome-wide networks related to mitochondria suggests that additional ASD genes related to mitochondria may remain to be discovered, and our hub gene analysis provides potential high confidence candidates.

While the phenotype of autism may ultimately result from dysfunctional synaptogenesis, it is possible that such fundamental cellular processes as protein translation, ubiquitination, alternative splicing, and mitochondrial function may underlie the synaptic dysfunction. Furthermore, this may help explain the incredibly variable clinical spectrum of autism and account for the increased prevalence of other complex medical problems in both the brain and other systems that ASD patients experience (Levy et al. 2009). Moreover, a recent metaanalysis of de novo mutations in autism demonstrated enrichment for genes related to transcriptional regulation and showed they have similar neurodevelopmental expression patterns to the green and blue modules of ASD candidates we identified (Ben-David and Shifman 2012a). Multiple recent whole-exome sequencing studies of individuals and family trios have continued to support the role of transcription- and synaptogenesis-related genes in ASD (Bernier et al. 2014; De Rubeis et al. 2014; Iossifov et al. 2014). Furthermore, a similar network analysis approach that assessed specifically for enrichment of de novo variants implicated in ASD and intellectual disability found similar shared transcriptional networks (Hormozdiari et al. 2015). By integrating co-expression and protein-protein interaction networks they demonstrated that ASD-related genes converge into two modules related to basic intracellular processes including transcriptional regulation and synaptogenesis, and that the former process was more operant in prenatal time periods and the later in postnatal development (Hormozdiari et al. 2015). These results are in line with earlier findings using either co-expression networks only (Parikshak et al. 2013, Willsey et al. 2013) or protein-protein interaction networks only (Pinto et al. 2014). Our results, despite assessing a much broader set of ASD candidate genes, are largely in agreement with these recent results. Whether and how defects in these basic cellular mechanisms result in altered synaptogenesis, are a reaction to altered synaptogenesis, or are mutually exclusive from synaptogenesis is unclear. However, our results in addition to these previous studies suggest that a complex interplay between these processes and synaptogenesis are related to each other through overlapping co-expression networks.

A number of studies have assessed for changes in gene expression in postmortem autistic brain directly (for a review, see, Lintas et al. 2012; Voineagu 2012). These studies have repeatedly shown that the autistic transcriptome is abnormally expressed compared with control brains across many different brain regions. The genes that are mis-expressed in autistic brains have been consistently demonstrated to be involved in pathways related to the synapse (Voineagu et al. 2011; Chow et al. 2012), immune response/apoptosis (Garbett et al. 2008; Voineagu et al. 2011; Chow et al. 2012), neurotransmitter receptors (Purcell et al. 2001), RNA splicing (Voineagu et al. 2011; Ziats and Rennert 2013; Chow et al. 2012), and mitochondrial function (Smith et al. 2012; Anitha et al. 2012b). These findings in autistic brain complement our results by showing that the ASD co-expression modules we discovered in the normal developing brain are functioning in the same pathways that are consistently disrupted in autistic brains.

Finally, the identified hub genes of ASD-enriched modules recapitulate the gene ontology analysis of these modules, strengthening the observation that basic cellular functions related to genome processing and mitochondrial function may represent a nexus in the genomic pathology of ASD. In addition, a number of hub genes relate to myeloid cells and apoptosis. There is a growing body of evidence implicating cytokine signaling, microglia-mediated synaptic pruning, and other immune-related processes in ASD (Maezawa et al. 2011), and this finding suggests the autism candidate genes may indirectly relate to processes that interact with these pathways through the transcriptional machinery. Furthermore, this supports our finding that the green module of autism candidate genes relates to apoptotic pathways. However, because 
comprehensive lists of microglia-specific marker genes are not available, we were unable to assess for enrichment of ASD candidate genes into this cell type in this study. By highlighting individual genes that are most central in the identified molecular interaction networks, the hub gene analysis may provide potential additional high-yield ASD candidates for their respective transcriptional networks.

In summary, we have profiled the transcriptional coexpression networks of autism candidate genes throughout normal human brain development to identify modules of ASD candidate genes with biologically relevant expression patterns. We have shown that these ASD modules are enriched for synaptogenesis, apoptosis, and GABA-ergic signaling, suggesting that pathways previously independently implicated in autism are related to each other through shared neurodevelopmental transcriptional networks. In addition, we expanded the analysis of ASD candidates to consider their relationship with the entire brain transcriptome. We demonstrated that ASD-enriched transcriptome-wide molecular interaction modules are related to mitochondrial function, splicing, and protein turnover, which suggests further ASD candidates related to these functions may remain to be discovered.

Our comprehensive analysis of the global co-expression relationships between ASD candidates demonstrates that the various pathways implicated in autism separately may relate to one another when considered in a broader functional genomics framework. Furthermore, our molecular interaction module analysis represents a valuable strategy to identify and prioritize other potential ASD candidate genes. Moreover, this approach can be used to assess genes from other complex neurodevelopmental and psychiatric disorders like schizophrenia, to uncover potential overlapping transcriptional pathways in the developing human brain among other gene sets.

\section{Material and Methods}

\section{Dataset Summary}

\section{Developing Human Brain Transcriptome Data}

We downloaded the BrainSpan transcriptional atlas from http://www.brainspan.org. Details of tissue acquisition and data processing can be found in the BrainSpan documentation. The atlas contains next-generation RNA sequencing (RNA-seq) data generated from 579 tissue samples. These samples were collected from 41 developing and adult postmortem brains of neurologically unremarkable donors spanning early pre-natal development ( 8 postconception weeks (PCW)) to late adulthood (40 years of age).

Some donor brains in the BrainSpan Atlas have missing data from certain brain regions. We excluded donors that had more than six regions missing. For donors with six or less missing regions, we imputed the data for the missing brain regions using a nearest neighbor approach. A full mathematical description of this is provided in the Supplementary Information. The resulting dataset contained 30 donor brains. From these donor brains, only the 16 brain regions that were present in all 30 donor datasets were analyzed. This filtration resulted in a final dataset derived from 30 donor brains across 16 brain regions, or 480 brain samples in total.

The 30 donor brains used in our analysis were further grouped into seven developmental stages according to the BrainSpan classification system (Fig. 1a). The transcriptomes of the cerebellar cortex (CBC), medial-dorsal nucleus of the thalamus (MD), striatum (STR), amygdala (AMY), hippocampus (HIP), and 11 areas of the neocortex (NCX) were assessed (Fig. 1b).

The resultant dataset contained RNA-seq expression values aligned to composite gene models, and given in units of reads per kilobase of exon model per million mapped reads (RPKM) (Mortazavi et al. 2008). Genes whose RPKM values were likely to represent noise rather than actual sequenced reads were discarded by removing any gene that did not have at least one expression value greater than or equal to five RPKM in any of the 480 tissue samples. The remaining set consisted of 13,563 genes expressed in the 30 donor samples assessed. The expression data was then normalized across all samples using quintile normalization. Finally, the data was $\log _{2}$-transformed for further analysis.

\section{ASD Gene List}

A comprehensive yet high confidence list of common ASD susceptibility genes (herein named "ASD list") was created by combining (taking the union) lists from three main ASD genes databases: AutDB (Basu et al. 2009), Autism Genetics Database (AGD) (Matuszek and Talebizadeh 2009), and AutKB484 (Xu et al. 2012) (a subset of AutKB determined by Xu et al. through ranking and scoring algorithm to be the most high-confidence ASD candidates). These databases each independently collected genes that have previously been associated with autism through a number of different experimental studies using various methods (namely GWAS, single-gene deletion syndromes that have autism as a component, genome-wide expression profiling, and genome-wide sequencing/CNV/linkage studies). ASD genes that were not present in the 13,563 genes we considered from the BrainSpan atlas (for instance, mitochondrially encoded genes) were discarded. The final ASD list consisted of 455 ASD susceptibility genes (Table S7).

\section{Co-expression of ASD Candidate Genes}

We calculated the Spearman's rank correlation between each pair of ASD candidate genes within each of the seven 
developmental stages separately. For each donor, the correlation between each gene pair is calculated across all 16 brain regions. The correlation between a gene pair in each developmental stage is the average of their correlation across all donors within the developmental stage. We focused our analysis on gene pairs with an absolute correlation value greater than 0.8 in at least one developmental stage (1168 out of 103,285 gene pairs). We used hierarchical clustering to cluster gene pairs using the Euclidean distance between the profiles and a complete linkage to merge clusters. Based on the heatmap of gene pair correlations across development, we cut the dendrogram to produce three clusters. The correlation pattern for each module was summarized by averaging all the gene pair correlation patterns included in the respective module.

\section{Transcriptome-Wide Co-expression Network}

We constructed a transcriptome-wide co-expression from all genes expressed in the brain (13,563 genes), based on the similarity of their expression profile across all samples (480 samples). We used hierarchical clustering to cluster gene pairs using Spearman's rank correlation between the profiles and a complete linkage to merge clusters. We cut the dendrogam to produce 32 modules of varying size (from 36 to 1386 genes)

\section{Gene Set Enrichment and Gene Ontology Enrichment Analysis}

Enrichment of transcriptome-wide molecular interaction modules for ASD candidate genes and cell-type-specific genes was assessed using the hypergeometric probability density function (hygepdf) in MATLAB R2011a (The MathWorks, Inc.). The resulting $p$ values were corrected for multiple testing using false discovery rate (FDR). All results reported are the $-\log 10$ of FDR-corrected $p$ values, and only $p$ values $<0.001$ were considered significant.

Gene list were assessed for shared biological pathways by testing for enrichment of gene ontology terms (GO) using DAVID Bioinformatics Resources 6.7 (Huang et al. 2009). The complete list of expressed genes in this study's dataset $(13,563$ genes) was used as the background. Only gene ontology terms with a Benjamini-Hochberg multiple testingcorrected $p$ value $<0.01$ are presented as significant.

Acknowledgments We gratefully acknowledge the Allen Institute for Brain Science. We thank Marc Hulsman for his input on data normalization. We also thank Silvère van der Maarel and Arn M.J.M. van den Maagdenberg for their critical insight on the manuscript. This research has received partial funding from the Dutch Technology Foundation STW, as part of the STW project 12721: "Genes in Space" under the IMAGENE perspective program, and from the European Union Seventh
Framework Program (FP7/2007-2013) under grant agreement no. 604102 (Human Brain Project).

Open Access This article is distributed under the terms of the Creative Commons Attribution 4.0 International License (http:// creativecommons.org/licenses/by/4.0/), which permits unrestricted use, distribution, and reproduction in any medium, provided you give appropriate credit to the original author(s) and the source, provide a link to the Creative Commons license, and indicate if changes were made.

\section{References}

Abrahams B, Tentler D, Perederiy J, et al (2007) Genome-wide analyses of human perisylvian cerebral cortical patterning. Proc Natl Acad Sci U S A 104:17849-17854. doi:10.1073/pnas.0706128104

American Psychiatric Association (2000) Diagnostic and statistical manual of mental disorders. American Psychiatric Association, Washington, D.C.

Anitha A, Nakamura K, Thanseem I, et al (2012a) Downregulation of the expression of mitochondrial electron transport complex genes in autism brains. Brain Pathol. doi:10.1111/bpa.12002

Anitha A, Nakamura K, Thanseem I, et al (2012b) Brain region-specific altered expression and association of mitochondria-related genes in autism. Mol Autism 3:12. doi:10.1186/2040-2392-3-12

Anney R, Klei L, Pinto D, et al (2010) A genome-wide scan for common alleles affecting risk for autism. Hum Mol Genet 19:4072-4082. doi:10.1093/hmg/ddq307

Bailey A, Le Couteur A, Gottesman I, et al (1995) Autism as a strongly genetic disorder: evidence from a British twin study. Psychol Med 25:63-77

Barabási A-L, Gulbahce N, Loscalzo J (2011) Network medicine: a network-based approach to human disease. Nat Rev Genet 12:5668. doi: $10.1038 / \mathrm{nrg} 2918$

Basu SN, Kollu R, Banerjee-Basu S (2009) AutDB: a gene reference resource for autism research. Nucleic Acids Res 37:6. doi:10.1093/ nar/gkn835

Ben-David E, Shifman S (2012a) Networks of neuronal genes affected by common and rare variants in autism spectrum disorders. PLoS Genet 8:e1002556. doi: 10.1371/journal.pgen.1002556

Ben-David E, Shifman S (2012b) Combined analysis of exome sequencing points toward a major role for transcription regulation during brain development in autism. Mol Psychiatry 18:1054-1056. doi: 10.1038/mp.2012.148

Berg JM, Geschwind DH (2012) Autism genetics: searching for specificity and convergence. Genome Biol 13:247. doi:10.1186/gb4034

Bernier R, Golzio C, Xiong B, et al (2014) Disruptive CHD8 mutations define a subtype of autism early in development. Cell 1-14. doi:10. 1016/j.cell.2014.06.017

Bourgeron T (2009) A synaptic trek to autism. Curr Opin Neurobiol 19: 231-234. doi:10.1016/j.conb.2009.06.003

Cahoy JD, Emery B, Kaushal A, et al (2008) A transcriptome database for astrocytes, neurons, and oligodendrocytes: a new resource for understanding brain development and function. J Neurosci 28:264 278. doi:10.1523/JNEUROSCI.4178-07.2008

Cao F, Yin A, Wen G, et al (2012) Alteration of astrocytes and Wnt $/ \beta$ catenin signaling in the frontal cortex of autistic subjects. J Neuroinflammation 9:223. doi:10.1186/1742-2094-9-223

Carper RA, Courchesne E (2005) Localized enlargement of the frontal cortex in early autism. Biol Psychiatry 57:126-133. doi:10.1016/j. biopsych.2004.11.005 
Centers for Disease Control and Prevention (2012) Prevalence of autism spectrum disorders - autism and developmental disabilities monitoring network, 14 sites, United States, 2008.

Chow ML, Pramparo T, Winn ME, et al (2012) Age-dependent brain gene expression and copy number anomalies in autism suggest distinct pathological processes at young versus mature ages. PLoS Genet 8:e1002592. doi: 10.1371/journal.pgen.1002592

Courchesne E, Pierce K (2005) Why the frontal cortex in autism might be talking only to itself: local over-connectivity but long-distance disconnection. Curr Opin Neurobiol 15:225-230. doi:10.1016/j.conb. 2005.03.001

De Rubeis S, He X, Goldberg AP, et al (2014) Synaptic, transcriptional and chromatin genes disrupted in autism. Nature. doi:10.1038/ nature 13772

Garbett K, Ebert PJ, Mitchell A, et al (2008) Immune transcriptome alterations in the temporal cortex of subjects with autism. Neurobiol Dis 30:303-311. doi:10.1016/j.nbd.2008.01.012

Geschwind DH (2011) Genetics of autism spectrum disorders. Trends Cogn Sci 15:409-416. doi:10.1016/j.tics.2011.07.003

Geschwind DH, Levitt P (2007) Autism spectrum disorders: developmental disconnection syndromes. Curr Opin Neurobiol 17:103111. doi:10.1016/j.conb.2007.01.009

Gkogkas C, Khoutorsky A, Ran I, et al (2013) Autism-related deficits via dysregulated eIF4E-dependent translational control. Nature 493: 371-377. doi:10.1038/nature11628

Glessner JT, Wang K, Cai G, et al (2009) Autism genome-wide copy number variation reveals ubiquitin and neuronal genes. Nature 459:569-573. doi:10.1038/nature07953

Hallmayer J, Cleveland S, Torres A, et al (2011) Genetic heritability and shared environmental factors among twin pairs with autism. Arch Gen Psychiatry 68:1095-1102. doi:10.1001/archgenpsychiatry. 2011.76

Hormozdiari F, Penn O, Borenstein E, Eichler EE (2015) The discovery of integrated gene networks for autism and related disorders. Genome Res 142-154. doi:10.1101/gr.178855.114.142

Huang DW, Sherman B, Lempicki R (2009) Systematic and integrative analysis of large gene lists using DAVID bioinformatics resources. Nat Protoc 4:44-57. doi:10.1038/nprot.2008.211

Huguet G, Ey E, Bourgeron T (2013) The genetic landscapes of autism spectrum disorders. Annu Rev Genomics Hum Genet. doi:10.1146/ annurev-genom-091212-153431

Iossifov I, O'Roak BJ, Sanders SJ, et al (2014) The contribution of de novo coding mutations to autism spectrum disorder. Nature. doi:10. 1038/nature13908

Iossifov I, Ronemus M, Levy D, et al (2012) De novo gene disruptions in children on the autistic spectrum. Neuron 74:285-299. doi:10.1016/ j.neuron.2012.04.009

Ip B, Wappler I, Peters H, et al (2010) Investigating gradients of gene expression involved in early human cortical development. J Anat 217:300-311. doi:10.1111/j.1469-7580.2010.01259.x

Johnson M, Kawasawa Y, Mason C, et al (2009) Functional and evolutionary insights into human brain development through global transcriptome analysis. Neuron 62:494-509. doi:10.1016/j.neuron. 2009.03.027

Kang HJ, Kawasawa YI, Cheng F, et al (2011) Spatio-temporal transcriptome of the human brain. Nature 478:483-489. doi:10.1038/ nature 10523

Kelleher R, Bear M (2008) The autistic neuron: troubled translation? Cell 135:401-406. doi:10.1016/j.cell.2008.10.017

Krumm N, O'Roak BJ, Shendure J, Eichler EE (2014) A de novo convergence of autism genetics and molecular neuroscience. Trends Neurosci 37:95-105. doi:10.1016/j.tins.2013.11.005

Levy S, Mandell D, Schultz R (2009) Autism. Lancet 374:1627-1638. doi:10.1016/S0140-6736(09)61376-3
Levy D, Ronemus M, Yamrom B, et al (2011) Rare de novo and transmitted copy-number variation in autistic spectrum disorders. Neuron 70:886-897. doi:10.1016/j.neuron.2011.05.015

Lintas C, Sacco R, Persico AM (2012) Genome-wide expression studies in autism spectrum disorder, rett syndrome, and down syndrome. Neurobiol Dis 45:57-68

Lioy D, Garg S, Monaghan C, et al (2011) A role for glia in the progression of Rett's syndrome. Nature 475:497-500. doi:10.1038/ nature 10214

Maezawa I, Calafiore M, Wulff H, Jin L-W (2011) Does microglial dysfunction play a role in autism and Rett syndrome? Neuron Glia Biol 7:85-97. doi:10.1017/S1740925X1200004X

Marshall C, Noor A, Vincent J, et al (2008) Structural variation of chromosomes in autism spectrum disorder. Am J Hum Genet 82:477488. doi:10.1016/j.ajhg.2007.12.009

Matuszek G, Talebizadeh Z (2009) Autism genetic database (AGD): a comprehensive database including autism susceptibility gene-CNVs integrated with known noncoding RNAs and fragile sites. BMC Med Genet 10:102. doi:10.1186/1471-2350-10-102

Mortazavi A, Williams B, McCue K, et al (2008) Mapping and quantifying mammalian transcriptomes by RNA-Seq. Nat Methods 5:621628. doi:10.1038/nmeth.1226

Neale BM, Kou Y, Liu L, et al (2012) Patterns and rates of exonic de novo mutations in autism spectrum disorders. Nature 485:242-245. doi: 10.1038/nature11011

Neves-Pereira M, Müller B, Massie D, et al (2009) Deregulation of EIF4E: a novel mechanism for autism. J Med Genet 46:759-765. doi:10.1136/jmg.2009.066852

O'Roak BJ, Vives L, Girirajan S, et al (2012) Sporadic autism exomes reveal a highly interconnected protein network of de novo mutations. Nature 485:246-250. doi:10.1038/nature10989

Oldham MC, Konopka G, Iwamoto K, et al (2008) Functional organization of the transcriptome in human brain. Nat Neurosci 11:12711282. doi:10.1038/nn.2207

Paolicelli RC, Bolasco G, Pagani F, et al (2011) Synaptic Pruning by Microglia Is Necessary for Normal Brain Development. Science (80-) 333:1456-1458. doi: DOI 10.1126/science.1202529

Parikshak NN, Luo R, Zhang A, et al (2013) Integrative functional genomic analyses implicate specific molecular pathways and circuits in autism. Cell 155:1008-1021. doi:10.1016/j.cell.2013.10.031

Pinto D, Delaby E, Merico D, et al (2014) Convergence of genes and cellular pathways dysregulated in autism spectrum disorders. Am J Hum Genet 94:677-94. doi: 10.1016/j.ajhg.2014.03.018

Pinto D, Pagnamenta A, Klei L, et al (2010) Functional impact of global rare copy number variation in autism spectrum disorders. Nature 466:368-372. doi:10.1038/nature09146

Piton A, Jouan L, Rochefort D, et al (2012) Analysis of the effects of rare variants on splicing identifies alterations in GABA(a) receptor genes in autism spectrum disorder individuals. Eur J Hum Genet. doi:10. 1038/ejhg.2012.243

Purcell AE, Jeon OH, Zimmerman AW, et al (2001) Postmortem brain abnormalities of the glutamate neurotransmitter system in autism. Neurology 57:1618-1628. doi:10.1212/WNL.57.9.1618

Ritvo E, Jorde L, Mason-Brothers A, et al (1989) The UCLA-University of Utah epidemiologic survey of autism: recurrence risk estimates and genetic counseling. Am J Psychiatry 146:1032-1036

Rossignol D, Frye R (2012) Mitochondrial dysfunction in autism spectrum disorders: a systematic review and meta-analysis. Mol Psychiatry 17:290-314. doi:10.1038/mp.2010.136

Rubenstein J, Merzenich M (2003) Model of autism: increased ratio of excitation/inhibition in key neural systems. Genes Brain Behav 2: 255-267. doi:10.1034/j.1601-183X.2003.00037.x

Sanders S, Ercan-Sencicek A, Hus V, et al (2011) Multiple recurrent de novo CNVs, including duplications of the 7q11.23 Williams syndrome region, are strongly associated with autism. Neuron 70:863885. doi:10.1016/j.neuron.2011.05.002 
Sanders SJ, Murtha MT, Gupta AR, et al (2012) De novo mutations revealed by whole-exome sequencing are strongly associated with autism. Nature 485:237-241. doi:10.1038/nature10945

Santini E, Huynh T, MacAskill A, et al (2013) Exaggerated translation causes synaptic and behavioural aberrations associated with autism. Nature 493:411-415. doi:10.1038/nature 11782

Sebat J, Lakshmi B, Malhotra D, et al (2007) Strong association of de novo copy number mutations with autism. Science 316:445-449. doi:10.1126/science.1138659

Sheikh A, Li X, Wen G, et al (2010) Cathepsin D and apoptosis related proteins are elevated in the brain of autistic subjects. Neuroscience 165:363-370. doi:10.1016/j.neuroscience.2009.10.035

Sheng Z-H, Cai Q (2012) Mitochondrial transport in neurons: impact on synaptic homeostasis and neurodegeneration. Nat Rev Neurosci 13: 77-93. doi:10.1038/nrn3156

Smalley S, Asarnow R, Spence M (1988) Autism and genetics. A decade of research. Arch Gen Psychiatry 45:953-961

Smith M, Flodman PL, Gargus JJ, et al (2012) Mitochondrial and ion channel gene alterations in autism. Biochimica et Biophysica ActaBioenergetics pp 1796-1802

Smith R, Sadee W (2011) Synaptic signaling and aberrant RNA splicing in autism spectrum disorders. Front Synaptic Neurosci 3:1. doi:10. 3389/fnsyn.2011.00001

Smoot M, Ono K, Ruscheinski J, et al (2011) Cytoscape 2.8: new features for data integration and network visualization. Bioinformatics 27: 431-432. doi:10.1093/bioinformatics/btq675

Somel M, Guo S, Fu N, et al (2010) MicroRNA, mRNA, and protein expression link development and aging in human and macaque brain. Genome Res 20:1207-1218. doi:10.1101/gr.106849.110

Spooren W, Lindemann L, Ghosh A, Santarelli L (2012) Synapse dysfunction in autism: a molecular medicine approach to drug discovery in neurodevelopmental disorders. Trends Pharmacol Sci 33:669684. doi:10.1016/j.tips.2012.09.004

Steffenburg S, Gillberg C, Hellgren L, et al (1989) A twin study of autism in Denmark, Finland, Iceland, Norway and Sweden. J Child Psychol Psychiatry 30:405-416. doi:10.1111/j.1469-7610.1989.tb00254.x

Stuart J, Segal E, Koller D, Kim S (2003) A gene-coexpression network for global discovery of conserved genetic modules. Science 302: 249-255. doi:10.1126/science.1087447
Sun T, Patoine C, Abu-Khalil A, et al (2005) Early asymmetry of gene transcription in embryonic human left and right cerebral cortex. Science 308:1794-1798. doi:10.1126/science.1110324

Szatmari P, Paterson AD, Zwaigenbaum L, et al (2007) Mapping autism risk loci using genetic linkage and chromosomal rearrangements. Nat Genet 39:319-328. doi: Doi 10.1038/Ng1985

Voineagu I (2012) Gene expression studies in autism: moving from the genome to the transcriptome and beyond. Neurobiol Dis 45:69-75. doi:10.1016/j.nbd.2011.07.017

Voineagu I, Wang X, Johnston P, et al (2011) Transcriptomic analysis of autistic brain reveals convergent molecular pathology. Nature 474: 380-384. doi:10.1038/nature10110

Wang K, Zhang H, Ma D, et al (2009) Common genetic variants on 5p14.1 associate with autism spectrum disorders. Nature 459:528 533. doi:10.1038/nature07999

Weiss L, Arking D, Gene Discovery Project of Johns Hopkins and the Autism Consortium, Daly MJ, Chakravarti A, et al (2009) A genome-wide linkage and association scan reveals novel loci for autism. Nature 461:802-808. doi:10.1038/nature08490

Willsey AJ, Sanders SJ, Li M, et al (2013) Coexpression networks implicate human midfetal deep cortical projection neurons in the pathogenesis of autism. Cell 155:997-1007. doi:10.1016/j.cell.2013.10. 020

Winden K, Oldham M, Mirnics K, et al (2009) The organization of the transcriptional network in specific neuronal classes. Mol Syst Biol 5: 291. doi:10.1038/msb.2009.46

Wolfe C, Kohane I, Butte A (2005) Systematic survey reveals general applicability of "guilt-by-association" within gene coexpression networks. BMC Bioinformatics 6:227. doi:10. 1186/1471-2105-6-227

Xu L-M, Li J-R, Huang Y, et al (2012) AutismKB: an evidence-based knowledgebase of autism genetics. Nucleic Acids Res 40:22. doi:10. 1093/nar/gkr1145

Ziats MN, Rennert OM (2013) Identification of differentially expressed microRNAs across the developing human brain. Mol Psychiatry 15. doi: $10.1038 / \mathrm{mp} .2013 .93$

Zoghbi H (2003) Postnatal neurodevelopmental disorders: meeting at the synapse? Science 302:826-830. doi:10.1126/science.1089071 